\title{
A Prototype Text to British Sign Language (BSL) Translation System
}

\author{
Ian Marshall, Éva Sáfár \\ School of Information Systems \\ University of East Anglia \\ im@sys.uea.ac.uk, es@sys.uea.ac.uk
}

\begin{abstract}
We demonstrate a text to sign language translation system for investigating sign language (SL) structure and assisting in production of sign narratives and informative presentations ${ }^{1}$. The system is demonstrable on a conventional PC laptop computer.
\end{abstract}

\section{Introduction}

During the last half century sign languages have been recognized as genuine languages. Thus sign languages are now accepted as minority languages, which coexist with majority languages (Neidle et al., 2000) and which are the native languages for many deaf people. Provision of information access and services in signed languages is as important as in other minority languages. Such provision, however, introduces theoretical and technical challenges. The use of a sign language gesture notation to drive virtual humans (avatars) for presenting signing has been investigated (Kennaway, 2001). Semi-automatic translation system from individual English sentences to such a sign language gesture notation has been demonstrated (self identifyinh references). Here, extension of this system to handle location of nominals at positions in the three dimensional space in front of the signer and noun verb agreement involving such allocated positions is described and illustrated.

\footnotetext{
${ }^{1}$ This work is incorporated within ViSiCAST, an EU Framework V supported project which builds on work supported by the UK Independent Television Commission and Post Office.
}

\section{Sign language phenomena}

Sign Languages (SLs) involve simultaneous manual and non-manual components for conveying meaning. Non-manual features are comprised of the posture of the upper torso, the orientation of the head and facial expressions. Manual features have been often been decomposed as hand-shape, hand orientation, hand position and motion (Stokoe, 1978; Brien, 1992; Sutton-Spence and Woll, 1999). The Hamburg Notation System (HamNoSys) (Prillwitz et al., 1989; Hanke and Schmaling, 2001; Hanke, 2002) is an established phonetic transcription system for SLs comprising more than 200 iconically motivated symbols to describe these manual and non-manual features of signs.

The manual components of signs are constrained to occur within signing space. Signing space is the three-dimensional space in front of the signer which extends vertically from above the signer's head to waist level, and horizontally from touching/close to the body to at arm's length in front of and to the side of the signer. Signs can be categorised in terms of the ways they use signing space. Body anchored and fixed nominal and verbal signs are either signed at a fixed body location or involve internal motion which allow relatively little modification to the sign. In contrast, some nominal signs can be signed at varying locations and thus the location where they are signed has significance. Furthermore, directional verbs allow grammatical and semantic information to be encoded within signing space such that the specific start and/or end positions of these signs have syntactic and semantic significance (Liddel, 1990). A further distinction can be made between topographic and syntactic use of space (Klima and Bel- 


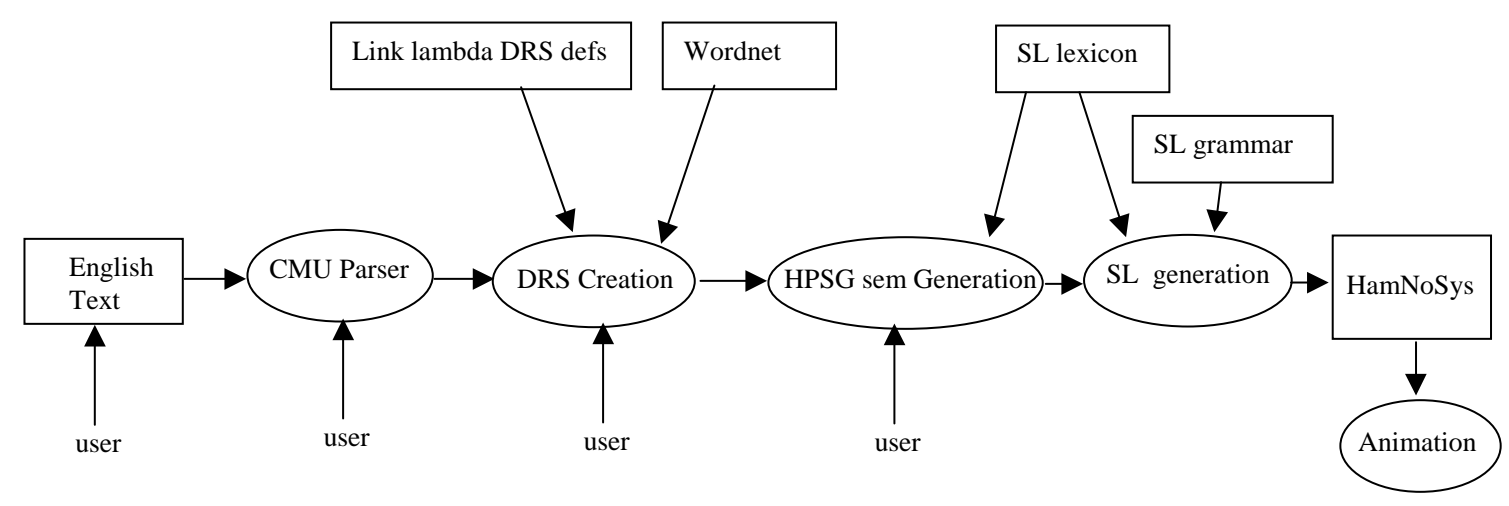

Figure 1: Architecture of the translation system

lugi, 1979; Emmorey et al., 1995; Sutton-Spence and Woll, 1999). In the case of the former, signing space is used to gesture towards and point at objects and persons physically present and thus has similarities with body anchored signs where the location at which a sign is made has an iconic/deictic function. However, in cases where the signer describes relationships between objects and persons which are not present, position within signing space can be used to denote abstract referents. Similarities between topographic and syntactic uses are apparent and often there is overlap between the two, and there is some evidence to suggest that, contrary to expectations, the granularity of the two may be comparable (Cormier, 2002). As our concerns are with translation from English text to sign language (and hence physical presence is not an issue) we concentrate on the syntactic uses of signing space.

\section{System Architecture}

The architecture of the English text to British Sign Langauge (BSL) system is essentially a pipeline of four main translation stages

1. English syntactic parsing,

2. Discourse Representation Structure (DRS) generation,

3. Semantic transfer,

4. Generation of HamNoSys SL phonetic descriptions,

as illustrated in Figure 1.

\subsection{Syntactic Parsing}

English text (Figure 2 top left) is parsed by the Carnegie Mellon University (CMU) link grammar parser (Sleator and Temperley, 1991) to produce an appropriate linkage which characterises syntactic dependencies (Figure 2 bottom left). In cases where multiple linkages are generated, the user intervenes to select an appropriate linkage.

\subsection{DRS Generation}

From a CMU parser generated linkage a Discourse Representation Structure DRS (Kamp and Reyle, 1993) is generated to capture the semantic content of the text (Figure 2 top middle). DRSs allow isolation of specific semantic content (nominal, verbal and adjectival based predicates, discourse referents and temporal relationships). Anaphora resolution is used to associate pronouns with discourse referents, and reuse of nouns is used to imply co-reference to the same linguistic referent. Currently, the most common 50\% CMU links are transformed into DRS form.

\subsection{Semantic Transfer}

An English oriented DRS is transformed into a SL oriented DRS. In particular, the number of arguments for some predicates is modified to a different number of arguments expected of a corresponding SL sign. For example, the English verb move obligatorily requires only one argument but is often accompanied by optional adjuncts for the source and destination locations. Its BSL equivalent (glossed as MOVE) requires three arguments - the start and end sign space positions and a (classifier or default) handshape consistent with the object being moved. Such transformations are effected on the DRS.

The DRS is then transformed to an equivalent 


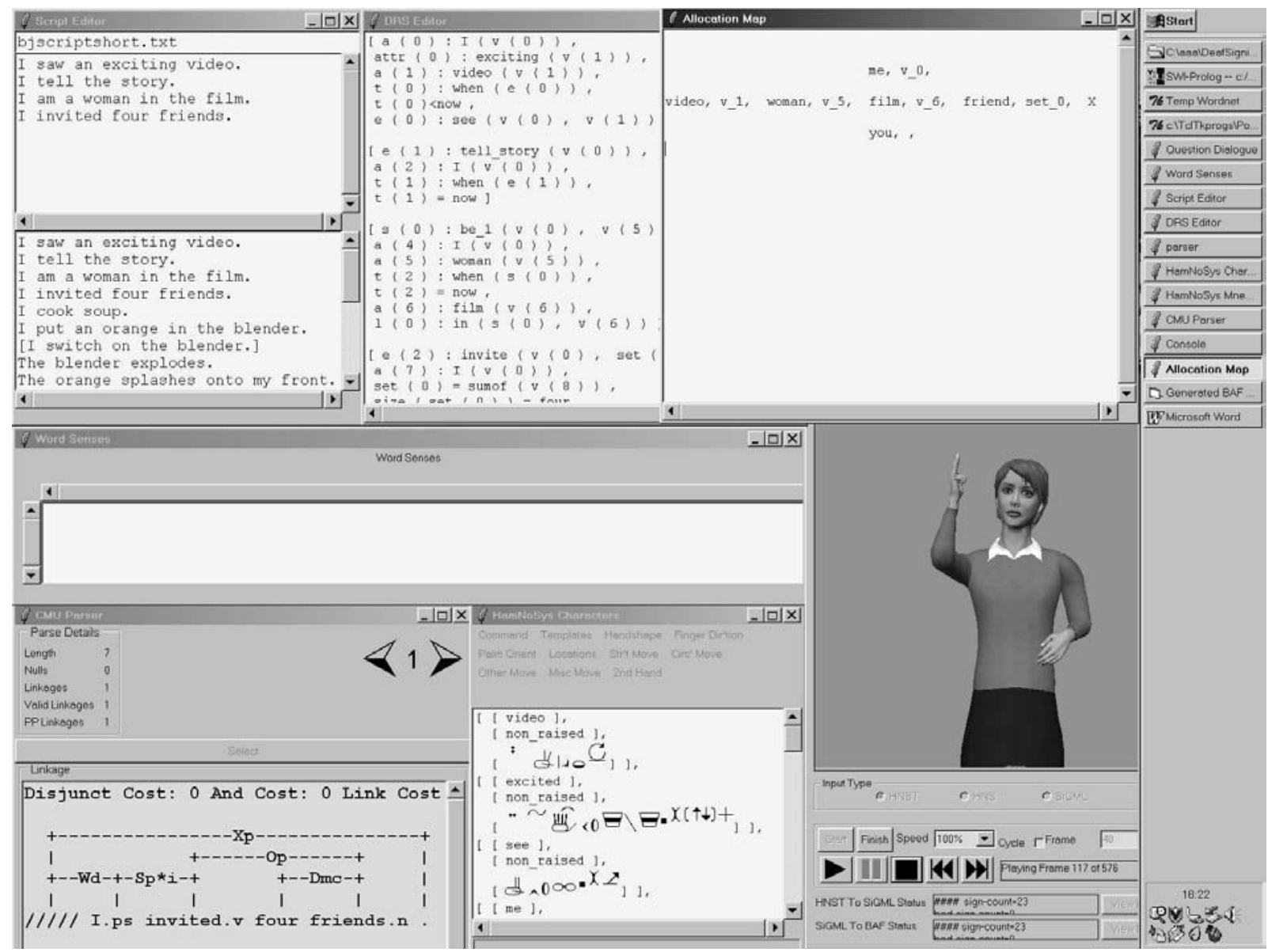

Figure 2: Screen shot of the current translation system

HPSG semantic structure which is the starting point for SL generation.

\subsection{HamNoSys SL Generation}

A SL grammar and lexicon are used to drive derivation of a HamNoSys phonetic description of a sign sequence from the HPSG semantic structure (Figure 2 bottom middle). The BSL lexicon contains approximately 250 lexical items. Some lexical items are fully instantiated forms for fixed and bodyanchored signs, however others are only partially instantiated forms for directional verbs and forms of modulation of lexical items. For nominal oriented signs, classifiers are associated with signs, and for directional verbs the lexical entries require incorporation of specific forms of classifiers and sign space locations.

The SL grammar constitutes a collection of simul- taneous constraints which the phonology and syntax of selected signs must satisfy in order to constitute a valid sign sequence. These constraints enforce appropriate sign order, for example realising a topic comment ordering signs for the English sentence " I saw an exciting video."

\section{VIDEO EXCITING/INTERESTING SEE ME}

Sign space location agreement requires that nominals are assigned consistent positions in signing space and that directional verbs agree with these positions that reflects anaphoric relationships of the original text and use with directional verbs. In this example, the directional verb SEE must start at the location of ME and be directed towards the location of VIDEO. Subsequent references to the same object must respect its position by signing the sign at the same location or by anaphoric pointing at that location. This form of agreement is achieved by in- 
clusion of a model of signing space within the HPSG feature structure in which nominals are allocated positions and from which verbal signs acquire positional information (Figure 2 top right).

Number agreement between nominal and verbal signs is enforced distinguishing between collective and distributive interpretations of plurals. For example, the friends in "I invited four friends" may have been invited individually (in which case the directional verb INVITE is repeated three times) or they may have been invited as a group (with INVITE signed only once). The under-specification in the English input is resolved by requesting the user to volunteer the additional information of a distributive or collective reading.

\section{Conclusions}

The resulting HamNoSys sign sequence descriptions are realised visually as virtual human behaviour (Kennaway, 2001) (Figure 2 bottom right) ${ }^{2}$. Currently, the SL generation sub-system incorporates a lexicon and grammar whose coverage are representative of a number of interesting SL phenomena and whose semantic, syntactic and phonological formalisation is one of the most advanced SL characterisations available. Such detail is essential to enable visualisation by a virtual human. The main omission in the system currently is the absence of nonmanual components of signing, though the SL generation has been designed to be extended in this direction in the future. The functionality of the system is demonstrable on a laptop computer.

\section{References}

D. (Ed.) Brien. 1992. Dictionary of British Sign Language/English. Faber and Faber, London,Boston.

K.A. Cormier. 2002. Grammaticization of indexic signs: How american sign language expresses numerosity. Doctoral thesis, Graduate School of the University of Texax at Austin.

K. Emmorey, D. Corina, and U. Bellugi. 1995. Differential processing of topographic and referential functions of space. In K. Emmorey and J.S. Reilly, editors, Language, Gesture, and Space, pages pp. 43-62. Lawrence Erlbaum Associates.

T. Hanke and C. Schmaling. 2001. A hamnosys-based phonetic transcription system as a basis for sign language generation. In Gesture Workshop 2001, London.

\footnotetext{
${ }^{2}$ The avatar illustrated was developed by Televirtual, Norwich UK and its HamNoSys interface by UEA colleagues within ViSiCAST.
}

T. Hanke. 2002. Hamnosys in a sign language generation context. In R. Schulmeister and H. Reinitzer, editors, Progress in sign language research.(In honor of Siegmund Prillwitz), International Studies on Sign Language and Communication of the Deaf; 40, pages pp. 249-264, Hamburg.

H. Kamp and U. Reyle. 1993. From Discourse to Logic. Introduction to Model theoretic Semantics of Natural Language, Formal Logic and Discourse Representation Theory. Kluwer Academic Publishers, Dordrecht.

J.R. Kennaway. 2001. Synthetic animation of deaf signing gestures. In The Fourth International Workshop on Gesture and Sign Language Interaction, Gesture Workshop 2001 (GW2001), City University, London, UK.

E. Klima and U. Bellugi. 1979. The signs of language. Harvard University Press.

S.K. Liddel. 1990. Structures for representing handshape and local movement at the phonemic level. In S.D. Fischer and P. Siple, editors, Theoretical Issues in Sign Language Research Vol 1, pages pp 37-65. University of Chicago Press.

I. Marshall and É. Sáfár. 2001. Extraction of semantic representations from syntactic cmu link grammar linkages. In G. Angelova, editor, Proceedings of Recent Advances in Natural Lanugage Processing, pages pp 154-159, Tzigov Chark, Bulgaria, Sept.

C. Neidle, J. Kegl, D. MacLaughlin, B. Bahan, and R.G. Lee. 2000. The Syntax of American Sign Language. MIT Press.

S. Prillwitz, R. Leven, H. Zienert, T. Hanke, J. Henning, et al. 1989. Hamburg Notation System for Sign Languages - An Introductory Guide. International Studies on Sign Language and the Communication of the Deaf, Volume 5., Institute of German Sign Language and Communication of the Deaf, University of Hamburg.

É. Sáfár and I. Marshall. 2001. The architecture of an englishtext-to-sign-languages translation system. In G. Angelova, editor, Recent Advances in Natural Language Processing (RANLP), pages pp223-228. Tzigov Chark, Bulgaria.

É. Sáfár and I. Marshall. 2002. Sign language translation via drt and hpsg. In A. Gelbukh (Ed.) Procieedings of the Third International Conference on Intelligent Text Processing and Computational Linguistics, CICLing, Mexico, Lecture Notes in Computer Science 2276, pages pp58-68, Springer Verlag, Mexico.

D. Sleator and D. Temperley. 1991. Parsing English with a Link Grammar. Carnegie Mellon University Computer Science technical report CMU-CS-91-196.

W.C. Stokoe. 1978. Sign language structure. (2nd ed.). Silver Spring, MD: Linstok Press.

R. Sutton-Spence and B. Woll. 1999. The Linguistics of British Sign Language. An Introduction. University Press, Cambridge. 